\title{
Changes of 1-deoxynojirimycin with Hypoglycemic Activity in Silkworm (Bombyx mori L.) During Different Developmental Stages
}

\author{
Hui-Juan Chen, Ying Shi, Jun Liu, Lixia Mu, Yu-Xiao Zou\# and Sen-Tai Liao* \\ Sericultural \& Agri-Food Research Institute, Guangdong Academy of Agricultural Sciences, Guangzhou, PR China
}

\begin{abstract}
Silkworms are drawing international scientific attention owing to their significant hypoglycemic activity, attributed to 1-deoxynojirimycin (DNJ), which they obtain from eating mulberry leaves. DNJ content in silkworms varies significantly during its life cycle as a holometabolic insect. The effect of metamorphosis and molting on DNJ accumulation remains unclear, however. More knowledge is needed about DNJ dynamics during the silkworm life cycle given their potential use as a functional food with hypoglycemic effect. This paper aims to elucidate the DNJ dynamics of silkworms over their life cycle. We propose the best physiological stage for silkworm harvesting in consideration of DNJ production. The effect of metamorphosis and molting on DNJ accumulation is also discussed. Samples from key physiological stages of silkworms were collected and the DNJ content analyzed by reverse-phase high-performance liquid chromatography (RP-HPLC). We observed fluctuations in DNJ levels depending on the physiological stage of the silkworm. A higher level of DNJ was found in the molted larvae than the molting ones. The highest DNJ level was detected in the molted larvae of instar III $(5.46 \mathrm{mg} / \mathrm{g})$ in terms of bodyweight, while a silkworm from Day 3 of instar V possesses the highest level of DNJ (1512.46 $\mu \mathrm{g}$ per silkworm). These results imply that natural physiological changes in the silkworm, including molt and metamorphosis, affect DNJ accumulation significantly. The period from Day 3 to Day 4 of the $5^{\text {th }}$ instar is considered the best time for silkworm harvesting given both DNJ concentration and silkworm biomass.
\end{abstract}

Keywords: 1-deoxynojirimycin; Hypoglycemic activity; a-glucosidase inhibitor; Developmental stages; Dynamics; Metamorphosis; Molting; Silkworm

\section{Introduction}

The burden of diabetes mellitus (DM) has been increasing globally over the last few decades. This condition is well known to impair vascular function and is associated with increased risk of cardiovascular diseases (CVD) [1]. Although numerous oral hypoglycemic drugs exist besides insulin, there is no promising therapy for DM [2]. The existing therapies are limited by their poor pharmacokinetic properties, secondary failure rates and accompanying side effects [3]. Current scientific evidence shows an adverse correlation between hyperglycemia and aggressive treatment with natural products possessing potent $\alpha$-glycosidase inhibitory activity [4-6]. Furthermore, the possibility of preventing the onset of diabetes using dietary supplements and/or herbal medicines has attracted considerable attention [7-9].

More recently, natural iminosugars are gathering increasing popularity in food formulation owing to their potential anti-diabetic effects. 1-Deoxynojirimycin (DNJ) is an iminosugar which was first isolated from mulberry trees [10]. It is a D-glucose analogue whereby an $\mathrm{NH}$ group substitutes for the oxygen atom of the pyranose ring, and it is well-known and highly effective a-glycosidase inhibitor (Figure 1). The chemical structure enables it to bind to carbohydrate digestive enzymes in the digestive tract and delay the digestion of the carbohydrate and its intestinal absorption. The competitive binding between DNJ and carbohydrates with the digestive enzymes leads to the delay of postprandial hyperglycemia. Therefore natural diet supplements with DNJ and other iminosugars possessing a-glucosidase inhibiting activity are of great interest.

The mulberry leaf is a valuable dietary resource of iminosugars. Besides DNJ, mulberry leaves are rich in other iminosugars including 2-O- $\alpha$-D-galactopyranosyl-1-deoxynojirimycin, $4-O-\beta$ D-glucopyranosyl-1-deoxynojirimycin, calystegine $B_{2}$, fagomine, 4-O- $\beta$-D-glucopyranosyl-fagomine, 3-epi-fagomine, 1,4-dideoxy1,4-imino-D-arabinitol, and 1,4-dideoxy-1,4-imino-(2- $O-\beta$-Dglucopyranosyl)-D-arabinitol. DNJ is the most abundant (making up $50 \%$ of the mulberry iminosugars) and the most powerful inhibitor of a-glucosidase among the compounds mentioned above [11]. However, DNJ levels in commercial mulberry leaf products is as low as only $0.1 \%$ of the dried mulberry leaf powder [12]. This suggests that the bioavailability of DNJ might not be sufficient through an oral intake of mulberry leaves. Therefore other natural resources whose DNJ levels are sufficient for controlling blood glucose are being explored.

Silkworms are oligophagous insects and exclusively eat mulberry leaves in the larval stage. Current scientific evidence demonstrates

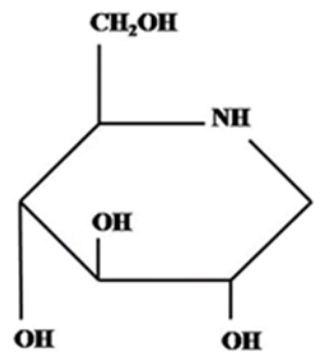

Figure 1: Chemical structure of 1-deoxynojirimycin.

*Corresponding authors: Sen-Tai Liao, Professor, Sericultural \& Agri-Food Research Institute, Guangdong Academy of Agricultural Sciences, NO.133 Yiheng St, Dongguanzhuang Rd, Tianhe District, Guangzhou 510610, China, Tel: +86-208759-6248; E-mail: liaost@163.com

\#Yu-Xiao Zou, Ph.D, Sericultural \& Agri-Food Research Institute, Guangdong Academy of Agricultural Sciences, NO.133 Yiheng ST, Dongguanzhuang RD Tianhe District, Guangzhou 510610, China, Tel: +86-20-37227141; E-mail: yuxiaozou@126.com

Received July 23, 2014; Accepted August 28, 2014; Published August 30, 2014

Citation: Chen HJ, Shi Y, Liu J, Mu L, Zou YX, et al. (2014) Changes of 1-deoxynojirimycin with Hypoglycemic Activity in Silkworm (Bombyx mori L.) During Different Developmental Stages. Med chem 4: 630-634. doi:10.4172/21610444.1000205

Copyright: $\odot 2014$ Chen HJ, et al. This is an open-access article distributed under the terms of the Creative Commons Attribution License, which permits unrestricted use, distribution, and reproduction in any medium, provided the original author and source are credited. 
that DNJ found in silkworms comes from the mulberry leaf [13]. Several studies in Korea have shown that silkworm powder and extract exhibited a significant hypoglycemic effect both in mice fed with a high carbohydrate-containing diet [14] and in alloxan-induced diabetic mice [15]. Another interesting result showed that DNJ can be concentrated several folds by silkworms feeding on mulberry leaves $[11,13,16]$. Silkworms have good potential as a functional food to improve blood glucose for subjects with glucose metabolism dysfunction.

The changes of DNJ during the complicated lifecycle of the silkworm has not been clearly characterized so far. The effects of complex physiological changes, e.g. exuviations and metamorphosis, on accumulation of DNJ are still limited. The best time for silkworm harvest with the purpose of gaining as much DNJ as possible has still not been fully elucidated. Silkworms from typical stages of exuviations and metamorphosis were collected to study DNJ dynamics depending on physiological stages regulated by hormones. We could then determine the optimal physiological stage with the highest DNJ level, which was quantified using HPLC.

\section{Materials and Methods}

\section{Reagents and chemicals}

Reference standard 1-deoxynojirimycin-HCl (DNJ-HCl) and fluorenyl-methyloxycarbonyl chloride (FMOC-Cl) were purchased from Sigma (St. Louis, MO, USA). Acetonitrile, methanol, acetic acid and other reagents were of analytical grade and were obtained from Tianjin Kermel Chemical Reagent Co., Ltd (Tianjin, China). The water used in the experiment was distilled and further purified by a Millipore Milli-Q Gradient system (MILLIPORE, USA).

\section{Sample preparation}

Liangguang 2 (LG 2), a widely fed silkworm cultivar in Southern China, was routinely fed in the laboratory by maintaining $80-85 \%$ relative humidity at $25^{\circ} \mathrm{C}$ according to a reported method [17]. Mulberry cultivar used for feeding silkworm is "Yuesang 11", and is classified as Morus atropurpurea Roxb., and the DNJ concentration was determined using HPLC $(0.38 \pm 0.03 \mathrm{mg} / \mathrm{g}$ dried mulberry leaves $)$. Developmental stages and corresponding numbers of 20 samples are shown in Table 1.

Silkworm behavior during metamorphosis is shown in Figure 2. Molting and molted larvae from larval instar I-V were collected to investigate the effect of exuviations on DNJ accumulation. Pupae and moths as well as their urine and feces were also collected to investigate the effect of metamorphosis on DNJ accumulation. Notably, the fourth molt only lasted for half a day, therefore the molting larvae of instar IV and molted larvae of instar V came from the same day. In total, 20 samples were lyophilized and pulverized for RP-HPLC analysis.

\section{Extraction of DNJ}

Extraction of DNJ from the silkworm was done according to the method of [18]. Briefly, $0.20 \mathrm{~g}$ of silkworm powder was extracted in 8 $\mathrm{mL}$ aqueous $0.05 \mathrm{M} \mathrm{HCl}$ by vortexing for $1 \mathrm{~min}$ at room temperature. Following centrifugation at $12000 \mathrm{rpm}$ for $15 \mathrm{~min}$, the supernatant was removed and the pellet was re-extracted twice more. The supernatants were combined and diluted to $25 \mathrm{~mL}$ with distilled water to obtain the silkworm powder extract (SPE).

\section{DNJ derivation}

Derivation of the DNJ standard or SPE was performed according the protocol of [18]. Briefly, $10 \mathrm{~mL} 0.4 \mathrm{M}$ potassium borate buffer $(\mathrm{pH}$ 8.5) was mixed with $10 \mu \mathrm{L} \mathrm{DNJ}$ standard or silkworm extracts, followed by the addition of $20 \mu \mathrm{L} 5 \mathrm{mM}$ FMOC-Cl in $\mathrm{CH}_{3} \mathrm{CN}$. The mixture was agitated and kept in a water bath at $20^{\circ} \mathrm{C}$ for $20 \mathrm{~min}$. Then $10 \mu \mathrm{L}$ $0.1 \mathrm{M}$ glycine was added to terminate the reaction by quenching the remaining FMOC-Cl. Finally, $950 \mu \mathrm{L}$ of $0.1 \%(v / v)$ aqueous acetic acid was added to stabilize the DNJ-FMOC and the mixture was filtered through a $0.22 \mu \mathrm{m}$ nylon syringe filter. A $10 \mu \mathrm{L}$ aliquot of the filtrate was injected into the HPLC system for analysis.

\section{DNJ quantification}

RP-HPLC was used for DNJ quantification according to the protocol described previously with minor modifications [18]. Analysis was conducted using a 1200 Series HPLC system (Agilent Technologies Inc., Karlsruhe, Germany) equipped with a Zorbax SB-C18 column $(250 \mathrm{~mm} \times 4.6 \mathrm{~mm}$ i.d., $5 \mu \mathrm{m})$ and an Agilent G1321A fluorescence detector (excitation $254 \mathrm{~nm}$, emission $322 \mathrm{~nm}$ ). The column was eluted with a mobile phase of acetonitrile: $0.1 \%(\mathrm{v} / \mathrm{v})$ aqueous acetic acid (40:60, v/v), pumped at $1.0 \mathrm{~mL} / \mathrm{min}$ for $20 \mathrm{~min}$. The column was kept at $25^{\circ} \mathrm{C}$, and the injection volume was $10 \mu \mathrm{L}$. DNJ concentrations were calculated based on the standard curves constructed with authentic standards. Concentration was expressed as $\mathrm{mg} / \mathrm{g}$ dried weight (DW) or mg per silkworm.

\section{Data analysis}

Statistical calculation was conducted using SPSS Statistics 17.0 (Version 17, SPSS Inc., Chicago, IL, USA, 2008). All results are expressed as mean \pm S.D. $(n=3)$. One-way ANOVA was performed to test difference between treatments within the same time points, followed by Tukey's HSD multiple comparison test, data without the same letters were considered significant $(p \leq 0.05)$.

\section{Results}

\section{DNJ Dynamics in silkworm during different developmental stages}

Food intake only happens during the larval stage of silkworms, and silkworms also stop feeding mulberry leaves for four times over the larval period. To elucidate the effect of molt and metamorphosis

\begin{tabular}{|c|c|c|c|c|c|}
\hline NO. & Sample & Time & NO. & Sample & Time \\
\hline 1 & newly-hatched larva & Day 1 & 11 & Day 2 of instar V & Day 17 \\
\hline 2 & molting larva of instar I & Day 3 & 12 & Day 3 of instar V & Day 18 \\
\hline 3 & molted larva of instar II & Day 4 & 13 & Day 4 of instar V & Day 19 \\
\hline 4 & molting larva of instar II & Day 6 & 14 & Day 5 of instar V & Day 20 \\
\hline 5 & molted larva of instar III & Day 7 & 15 & Day of instar V & Day 21 \\
\hline 6 & molting larva of instar III & Day 10 & 16 & matured larva V & Day 22 \\
\hline 7 & molted larva of instar IV & Day 11 & 17 & Day 1 of cocooning & Day 23 \\
\hline 8 & molting larva of instar IV & Day 15 & 18 & Day 2 of cocooning & Day 24 \\
\hline 9 & molted larva of instar V & Day 15 & 19 & pupa & Day 26 \\
\hline 10 & Day 1 of instar V & Day 16 & 20 & moth (adult) & Day 36 \\
\hline
\end{tabular}

Note: "Sample" means the physiological stage of the silkworm, while "Time" means the corresponding time point during the whole life cycle of silkworm. No. 8 and No. 9 were collected at 8:00 and 18:00 of the same day, respectively.

Table 1: Silkworm samples and corresponding developmental stages.

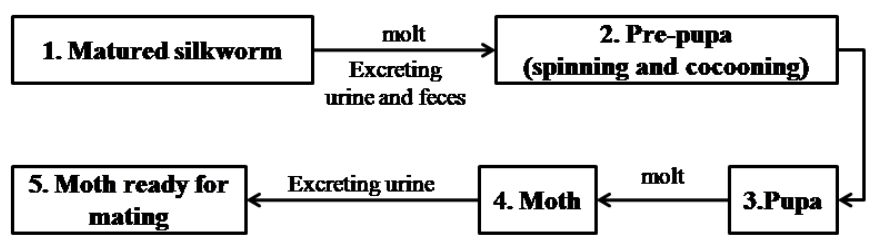

Figure 2: Physiological process of silkworm from matured larvae to moths. 
on DNJ accumulation in silkworms, we collected typical molting and molted samples from instars, as well as pupae and moths etc. Plotting DNJ levels in in silkworm by developmental stage gave a fluctuant curve with some degree of regularity (Figure 3). DNJ was detected in every sample except for the newly hatched larvae and in the moth. The larval stage was found to be the primary period for DNJ accumulation compared to the other three stages.

We first detected DNJ in molting larvae $(4.02 \mathrm{mg} / \mathrm{g})$ after 2 days of mulberry leaf feeding. After a one-day molt, a minor elevation was observed in molted larva II $(4.07 \mathrm{mg} / \mathrm{g})$. Interestingly, we found that DNJ content in larvae tended to increase after a transient molt initiated by hormones, e.g. elevations were observed around 4 molts during the larval stage. While within instar III and VI, DNJ in molted larvae was higher than that in molting larvae. The maximal DNJ level $(5.46 \mathrm{mg} / \mathrm{g})$ was reached at the beginning of instar III, namely, molted larvae of instar III. The next highest DNJ level was observed at the molted larvae of the instar VI $(5.14 \mathrm{mg} / \mathrm{g})$ and then at the molted larvae of instar $\mathrm{V}(4.39 \mathrm{mg} / \mathrm{g})$. Silkworms get to a bodyweight peak at instar $\mathrm{V}$, and within this instar, DNJ in the sample at Day 3 reaches a maximum value at $3.43 \mathrm{mg} / \mathrm{g}$, followed by a sharp decrease from Day 4 to the matured larvae and a persistent low until the moth stage (Figure 3).

\section{DNJ distribution during metamorphosis}

The metamorphosis of silkworms is governed by ecdysteroids and juvenile hormones, a more complicated process than larval molt [19]. It is usually coupled with urine excretion, spinning and cocooning as well as molting (Figure 2). Silkworms stop feeding after the larval stage and so exogenous DNJ from the mulberry leaves is not available after the silkworms have matured. Therefore we monitored DNJ in matured silkworms, pupae and moths, as well as their feces and urine, to elucidate the distribution and movement of DNJ.

DNJ in matured larvae begins to obviously drop off after the 6th day of instar V and is nearly undetectable in moths (Figure 3). During this period, the silkworms do not take in mulberry leaves, but are excreting, so the lost DNJ is expected to be in the excrement. As shown in Table 2, matured larvae I that had partially excreted urine and feces were found with DNJ levels of $0.30 \pm 0.03 \mathrm{mg} / \mathrm{g}$. With more waste excreted out of the silkworm, a consequent lower DNJ level was found in matured larvae II $(0.13 \pm 0.01 \mathrm{mg} / \mathrm{g})$. DNJ levels in the urine and feces were $0.0044 \pm$ $0.0031 \mathrm{mg} / \mathrm{g}$ and $0.96 \pm 0.06 \mathrm{mg} / \mathrm{g}$, respectively. The feces of the larvae contain a large amount of undigested mulberry leaves, giving higher levels of DNJ even than in the larvae. We observed a small increase in the amount of DNJ in both the pupae and the moths before excreting urine, compared with in the matured larvae II. This is probably owing to the complicated nutrient changes during metamorphosis such as water loss and fat synthesis etc. [20,21]. DNJ was not detected in the moths, indicating they are not suitable as an edible resource of DNJ.

\section{Analysis of DNJ based on the individual weigh of the silkworm}

Silkworms go through a huge weight change over their life cycle. Analysis of DNJ production based on the individual weight of silkworms and corresponding DNJ concentration was performed to establish the best stage to use as a functional food. Although the molted larvae of instar III possess the highest level of DNJ, there is a 20 -fold weight difference between matured larvae (instar V) and the molted larva of the 3rd instar. Therefore, the total DNJ production contributed by the molted larva from instar III is much less than that of the matured larvae of instar V. As shown in Table 3, the contents of DNJ in individual silkworms increased continuously with the development of the silkworm. It reaches its highest concentration in the $4^{\text {th }}$ day of instar V (1512.46 $\mu \mathrm{g} /$ per silkworm), followed by the $3^{\text {rd }}$ day of instar $\mathrm{V}$
(1499.39 $\mu \mathrm{g} /$ per silkworm). From then on DNJ begins to decrease and reaches zero on the 2 nd day after cocooning.

\section{Discussion}

\section{DNJ in silkworms comes from mulberry leaves}

The mulberry leaf is a well-known edible resource of DNJ with hypoglycemic activity [11]. Silkworms are oligophagous insects that feed exclusively on this plant. We did not find DNJ in newly hatched larvae, but first detected it in the molting larva of instar I after two days of mulberry leaf intake. This result is consistent with that of Yin $\mathrm{H}$ et al. [13], who reported that DNJ was not detected in silkworms fed with artificial feed without mulberry leaf powder, except in newly hatched larvae. Furthermore, the results from later samples in mature silkworms showed that DNJ levels went down sharply and diminished until the moth stage as feeding on the mulberry leaf had stopped. It is therefore obvious that the DNJ found in silkworms comes from mulberry leaves. $\mathrm{DNJ}$ content remained at a relatively high and more stable level during

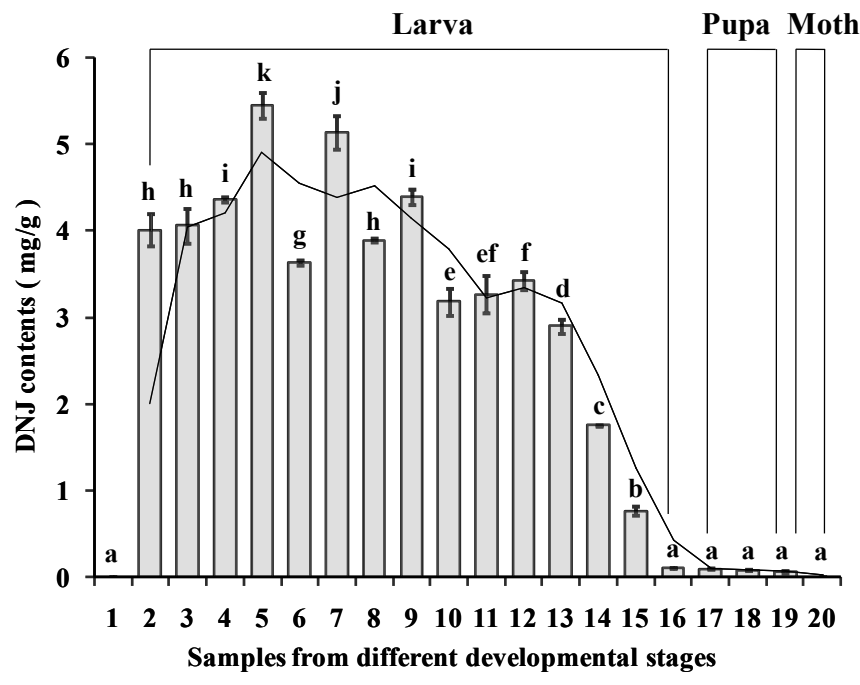

Figure 3: DNJ dynamics during the different developmental stages of the silkworm. Note: 20 samples were taken from different developmental stages and numbered as follow: 1 . Newly hatched larvae; 2 . Molting larvae of instar I; 3. Molted larvae of instar II; 4. Molting larva of instar II; 5 . Molted larva of the instar III; 6. Molting larvae of instar III; 7. Molted larvae of instar IV; 8. Molting larvae of instar IV; 9 . Molted larvae of instar V; 10. Day 1 of instar V; 11. Day 2 of instar V; 12. Day 3 of instar V; 13. Day 4 of instar V; 14. Day 5 of instar V; 15. Day 6 of instar $V ; 16$. Matured larvae; 17. Day 1 of cocooning; 18. Day 2 of cocooning; 19. Pupae; 20. Moth. Values are expressed as the mean \pm S.D. $(n=3)$. Means without the same letters were considered significant, tested by one-way ANOVA and Fisher's LSD, $p \leq 0.05$.

\begin{tabular}{|c|c|c|}
\hline No. & Sample & DNJ $(\mathrm{mg} / \mathrm{g})$ \\
\hline 1 & matured larvae I & $0.30 \pm 0.030 \mathrm{~d}$ \\
\hline 2 & matured larvae II & $0.13 \pm 0.010 \mathrm{~b}$ \\
\hline 3 & urine of matured larvae & $0.0044 \pm 0.0031 \mathrm{a}$ \\
\hline 4 & feces of matured larvae & $0.96 \pm 0.060 \mathrm{e}$ \\
\hline 5 & pupae & $0.23 \pm 0.020 \mathrm{c}$ \\
\hline 6 & moths before excreting urine & $0.24 \pm 0.040 \mathrm{c}$ \\
\hline 7 & urine of moths & $0.029 \pm 0.013 \mathrm{a}$ \\
\hline 8 & moths after excreting urine & ND a \\
\hline
\end{tabular}

Note: Matured larvae I means that the matured larvae have not excreted all the urine and excrement; Matured larvae II means that the matured larvae have excreted all the urine and excrement and have begun silking; ND: not detected. Values are expressed as the means $\pm S D(n=3)$. Means without the same letters were considered significant, tested by one-way ANOVA and Fisher's LSD, $p \leq 0.05$.

Table 2: DNJ concentration of samples from matured larvae to moths. 


\begin{tabular}{|c|c|c|c|}
\hline \hline Developmental stages & DNJ $(\mu \mathrm{g} /$ per silkworm $)$ & Developmental stages & DNJ $(\mu \mathrm{g} / \mathrm{per}$ silkworm $)$ \\
\hline newly hatched larvae & $0.00 \mathrm{a}$ & 1st day of instar V & $468.62 \pm 13.53 \mathrm{i}$ \\
\hline molting larvae of instar I & $3.28 \pm 0.56 \mathrm{a}$ & 2nd day of instar V & $710.34 \pm 15.41 \mathrm{k}$ \\
\hline molted larvae of instar II & $2.91 \pm 0.38 \mathrm{a}$ & 3rd day of instar V & $1499.39 \pm 20.13 \mathrm{~m}$ \\
\hline molting larvae of instar II & $19.12 \pm 1.22 \mathrm{~b}$ & 4th day of instar V & $1512.46 \pm 25.68 \mathrm{n}$ \\
\hline molted larvae of instar III & $19.23 \pm 1.09 \mathrm{~b}$ & 5th day of instar V & $990.00 \pm 6.85 \mathrm{I}$ \\
\hline molting larvae of instar III & $61.30 \pm 2.87 \mathrm{e}$ & 6th day of instar V & $484.55 \pm 12.66 \mathrm{ij}$ \\
\hline molted larvae of instar IV & $80.63 \pm 3.49 \mathrm{~g}$ & matured larvae & $68.51 \pm 3.01 \mathrm{f}$ \\
\hline molting larvae of instar IV & $393.36 \pm 10.26 \mathrm{~h}$ & 1st day of cocooning & $39.71 \pm 1.42 \mathrm{~d}$ \\
\hline molted larvae of instar V & $392.72 \pm 12.45 \mathrm{~h}$ & 2nd day of cocooning & $26.35 \pm 0.98 \mathrm{c}$ \\
\hline
\end{tabular}

Note: Means without the same letters were considered significant, tested by one-way ANOVA and Fisher's LSD, $p \leq 0.05$.

Table 3: DNJ content of individual silkworm during different developmental stages.

the larval stage compared to that of pupal and adult stages. Moreover, $\mathrm{DNJ}$ can be concentrated by silkworm through eating mulberry leaves. In this study, we found DNJ was elevated 9.03 and 14.37 -fold by silkworm at molted larva of the instar III, and Day 3 of instar V in terms of silkworm powder weigh, respectively. The difference of DNJ concentration may result from the different cultivar of silkworm and mulberry. Hence, the larval stage is the key physiological stage for DNJ accumulation compared to the pupal and moth stages.

\section{Effect of molt during larval stage on DNJ accumulation}

Molting is a hormonally initiated process in which the old skin is cast off and a new, larger one is produced, causing the larvae to go into the next instar. This eating-molting cycle repeats four times during the larval stages. The five successive larval instars are behaviorally and morphologically separated by ecdysis (molting). After hatching from the egg, larvae keep feeding on mulberry leaves except during the molting period. The dynamics of DNJ during the larval stage has not been extensively studied and the effect of molt on DNJ levels is unknown. Korean scientists used silkworms from Day 3 of instar V to treat diabetic mice and demonstrated its antihyperglycemic effect [15]. Yin et al. [13] reported DNJ contents in the silkworm at different developmental stages and tissues. However, the effect of molt during larval stage on DNJ accumulation was still not well understood. In this study we specifically added more sampling points before and after each molt to give a more comprehensive data set compared to previous studies $[13,16]$. We demonstrated that molting affected DNJ contents significantly. According to our data, DNJ tends to increase after a molt, e.g., the molted larvae of instar II > molting larvae of instar I and the molted larvae of instar III > molting larvae of instar II. As we mentioned above, mulberry leaves are the only DNJ resource for silkworms, and they stop feeding at the beginning of every molt. We speculated two possible reasons for the increased DNJ levels in the molted larvae. First, loss of water during molting [20] leads to a relative increase in DNJ content compared to the sample before molting. Another reason could be transformation of DNJ analogues from mulberry leaves, such as $\mathrm{N}$-methyl-DNJ, fagomine, 3-epi-fagomine and 2-o- $\alpha$-D-synthesis-1$\mathrm{DNJ}$ during the physiological change, coupled with complex biomarker transformations triggered by ecdysone [11].

\section{Effect of metamorphosis on DNJ accumulation}

Metamorphosis is a complex process that includes hyperplastic and hypertrophic growth as well as apoptosis and histolysis of specific larval tissues [20]. In this study, we demonstrated that DNJ levels went down sharply and diminished until the moth stage as soon as metamorphosis started. This result is in agreement with previous studies $[13,16]$. We detected DNJ in the waste excreted by mature larvae and pupae that were ready for metamorphosis (Table 2). Therefore, the loss of DNJ is caused by specific physiological behavior such as excreting urine and feces.

\section{Harvest time for silkworms in consideration of DNJ}

Silkworms develop very rapidly from age to age and increase by several times their original weight and size in each instar. The rate of increase varies from hatching to instar I, 4 to 5 times each at instar II and III, and 25 times each at instar IV and V. The total increase in weight from hatching to the end of the instar V will be between 7000-10,000 times. The appetite increases with the age of the larvae. Of the total ingestion during the entire larval period, nearly $85 \%$ is taken during instars IV and V. We analyzed DNJ content in two ways, one in terms of weight and another in terms of individual silkworms. Although the molted larvae of instar III were found to have the highest level of DNJ among the 20 samples. DNJ analysis in terms of individual silkworms showed that it would be more cost-effective if we collect the larvae of instar $\mathrm{V}$, when considering total DNJ production.

From the aspect of the unit weight of silkworm powder, the DNJ content reached its highest level in the molted larvae of instar III, and this may result in a better effect when taking it medicinally as a hypoglycemic functional food. However, from the aspect of individual silkworms, the content of DNJ reached its highest level in the 4th day of instar V (1512.46 $\mu \mathrm{g} /$ per silkworm), followed by the 3rd day of instar $\mathrm{V}$ (1499.39 $\mu \mathrm{g} /$ per silkworm). DNJ content increased by 77.66 times and 76.98 times per individual silkworm when compared with the molted larvae of instar III. In short, the silkworm of $3^{\text {rd }}$ and $4^{\text {th }}$ day of instar V should be selected as the best harvest time for silkworms rich in DNJ.

\section{Conclusion}

Our data shows that the silkworm is a good edible resource of natural DNJ with hypoglycemic activity. Molting and metamorphosis were shown to significantly affect DNJ accumulation in silkworms throughout their lifecycle. The 3rd and 4th day of instar $\mathrm{V}$ are recommended as the best harvest times when considering total DNJ production.

\section{Acknowledgement}

We sincerely thank Yang Xiao for her kind help in providing samples and species/cultivar information.

Funding sources: This work was supported by the Special Fund for Agroscientific Research in the Public Interest, China (No.201403064), Key Projects in the National Science \& Technology Pillar Program during the Twelfth Five-year Plan Period, China (No.2013BAD16B09), and Foundation of President of the Guangdong Academy of Agricultural Sciences (No. 201433).

\section{References}

1. Blaak EE, Antoine JM, Benton D, Björck I, Bozzetto L, et al. (2012) Impact of postprandial glycaemia on health and prevention of disease. Obes Rev 13: 923-984.

2. Ghosh S, Suryawanshi SA (2001) Effect of Vinca rosea extracts in treatment of alloxan diabetes in male albino rats. Indian J Exp Biol 39: 748-759. 
Citation: Chen HJ, Shi Y, Liu J, Mu L, Zou YX, et al. (2014) Changes of 1-deoxynojirimycin with Hypoglycemic Activity in Silkworm (Bombyx mori L.) During Different Developmental Stages. Med chem 4: 630-634. doi:10.4172/2161-0444.1000205

3. Melander A (1988) Sulphonylureas in the treatment of non-insulin-dependent diabetes. Baillieres Clin Endocrinol Metab 2: 443-453.

4. Huang Q, Chen S, Chen H, Wang Y, Wang Y, et al. (2013) Studies on the bioactivity of aqueous extract of pu-erh tea and its fractions: in vitro antioxidant activity and a-glycosidase inhibitory property, and their effect on postprandial hyperglycemia in diabetic mice. Food Chem Toxicol. 53: 75-83.

5. Hou W, Li Y, Zhang Q, Wei X, Peng A, et al. (2009) Triterpene acids isolated from Lagerstroemia speciosa leaves as alpha-glucosidase inhibitors. Phytother Res 23: 614-618.

6. Yatsunami K, Ichida M, Onodera S (2008) The relationship between 1-deoxynojirimycin content and alpha-glucosidase inhibitory activity in leaves of 276 mulberry cultivars (Morus spp.) in Kyoto, Japan. J Nat Med 62: 63-66.

7. Kimura T, Nakagawa K, Kubota H, Kojima Y, Goto Y, et al. (2007) Food-grade mulberry powder enriched with 1-deoxynojirimycin suppresses the elevation of postprandial blood glucose in humans. J Agric Food Chem 55: 5869-5874.

8. Liu CY, Huang CJ, Huang LH, Chen IJ, Chiu JP, et al. (2014) Effects of green tea extract on insulin resistance and glucagon-like peptide 1 in patients with type 2 diabetes and lipid abnormalities: a randomized, double-blinded, and placebo-controlled trial. PLoS One 9: e91163.

9. Ye EQ, Chacko SA, Chou EL, Kugizaki M, Liu S (2012) Greater whole-grain intake is associated with lower risk of type 2 diabetes, cardiovascular disease, and weight gain. J Nutr 142: 1304-1313.

10. Yagi M, Kouno T, Aoyagi Y, Murai H (1976) The structure of Moraoline, a piperidine alkaloid from Morus species. Nippon Nougei Kagaku Kaishi, 50: $571-572$.

11. Asano N, Yamashita T, Yasuda K, Ikeda K, Kizu H, et al. (2001) Polyhydroxylated alkaloids isolated from mulberry trees (Morusalba L.) and silkworms (Bombyx mori L.). J Agric Food Chem 49: 4208-4213.

12. Kimura T, Nakagawa K, Saito Y, Yamagishi K, Suzuki M, et al. (2004) Determination of 1-deoxynojirimycin in mulberry leaves using hydrophilic interaction chromatography with evaporative light scattering detection. J Agric Food Chem 52: 1415-1418.

13. Yin $H$, Shi $X Q$, Sun $B$, Ye JJ, Duan $Z A$, et al. (2010) Accumulation of 1-deoxynojirimycin in silkworm, Bombyx mori L. J Zhejiang Univ Sci B 11: 286291.

14. Chung S, Kim MS, Ryu KS (1997) Effect of silkworm extract on intestinal R-glucosidase activity in mice administrated with a high carbohydratecontaining diet. Korean J Seric Sci 39: 86-92.

15. Ryu KS, Lee HS, Chung SH, Kang PD (1997) An activity of lowering bloodglucose levels according to preparative conditions of silkworm powder. Korean J Seric Sci 39: 79-85.

16. Liu YQ, Ouyang Z, Zhao M, Chen HX, Pan G, et al. (2013) Correlational Study on 1-Deoxynojirimycin Content in Mulberry Leaves and Its Enrichment in Different Growth Stage of Silkworm Larva, Bombyx mori. Nat Prod Res Dev 25: $960-962$.

17. Ramesha C, Seshagiri SV, Rao CGP (2009) Evaluation and Identification of Superior Polyvoltine Crossbreeds of Mulberry Silkworm, Bombyx mori L. Journal of Entomology 6: 188-197.

18. Kim JW, Kim SU, Lee HS, Kim I, Ahn MY, et al. (2003) Determination of 1-deoxynojirimycin in Morus alba L. leaves by derivatization with 9-fluorenylmethyl chloroformate followed by reversed-phase high-performance liquid chromatography. J Chromatogr A 1002: 93-99.

19. Gilbert LI, Rybczynski R, Tobe SS (1996) Endocrine cascade in insect metamorphosis. In Metamorphosis: Postembryonic Reprogramming of Gene Expression in Amphibian and Insect Cells. Academic Press: New York.

20. Blossman-Myer BL, Burggren WW (2010) Metabolic allometry during development and metamorphosis of the silkworm Bombyx mori: analyses, patterns, and mechanisms. Physiol Biochem Zool 83: 215-231.

21. Yoshio H, Shinkichi S (1989) Two new phenolic compounds, kuwanols $C$ and $\mathrm{D}$, from the root bark of a Mulberry tree. Heterocycles 29: 807-813. 